\title{
A comparison of field-line resonances observed at the Goose Bay and Wick radars
}

\author{
G. Provan, T. K. Yeoman \\ Department of Physics and Astronomy, University of Leicester, University Road, Leicester LE1 7RH, UK
}

Received: 3 January 1996 / Revised: 10 June 1996 / Accepted: 30 July 1996

\begin{abstract}
Previous observations with the Goose Bay HF coherent-scatter radar have revealed structured spectral peaks at ultra-low frequencies. The frequencies of these spectral peaks have been demonstrated to be extremely consistent from day to day. The stability of these spectral peaks can be seen as evidence for the existence of global magnetospheric cavity modes whose resonant frequencies are independent of latitude. Fieldline resonances occur when successive harmonics of the eigenfrequency of the magnetospheric cavity or waveguide match either the first harmonic eigenfrequency of the geomagnetic field lines or higher harmonics of this frequency. Power spectra observed at the SABRE VHF coherent-scatter radar at Wick, Scotland, during night and early morning are revealed to show similarly clearly structured spectral peaks. These spectral peaks are the result of local field-line resonances due to Alfvén waves standing on magnetospheric field lines. A comparison of the spectra observed by the Goose Bay and Wick radars demonstrate that the frequencies of the field-line resonances are, on average, almost identical, despite the different latitudinal ranges covered by the two radars. Possible explanations for the similarity of the signatures on the two radar systems are discussed.
\end{abstract}

\section{Introduction}

Field-line resonances are often detected with both ground-based magnetometers and radar data. These pulsations in the magnetic and electric field are characterised by having a phase change of $180^{\circ}$ in latitude across the region of maximum amplitude on the ground. The resonances are a consequence of standing Alfvén waves on geomagnetic field lines, and the wavelength of these Alfvén waves is such that an integral number of half wavelengths fits into the magnetic flux

Correspondence to: $\mathrm{G}$. Provan tube linking the northern and southern hemisphere (see e.g. Hughes, 1983, and references therein)

Dungey (1954) first suggested that the origin of fieldline resonances was the Kelvin-Helmholtz instability at the magnetopause boundary. These instabilities would create compressional surface waves in the outer magnetosphere. In the presence of inhomogeneities in the magnetoplasma, these compressional waves couple to transverse Alfvén waves of appropriate eigenperiods and hence magnetospheric field lines would be driven at their resonant periods (Tamao, 1965; Southwood, 1974; Chen and Hasegawa, 1974).

This theory proved successful in explaining many of the observed features of field-line resonances. However, in these early theories there was no explanation as to why, despite the presence of low-level power at a broad range of ultra-low frequencies in the magnetosphere, the observed field-line resonances were often dominated by discrete frequencies. This difficulty led Kivelson and Southwood (1985) to suggest that a resonant cavity could form in the magnetosphere within which the frequencies of the compressional waves are quantised. The boundaries of the cavity were suggested as being the magnetopause and the turning points of the compressional waves on dipole-like field lines in the inner magnetosphere.

Some of the strongest experimental evidence for the existence of magnetospheric cavities has come from the inner magnetosphere, the plasmasphere. For example Sutcliffe and Yumoto (1989, 1991), Yeoman and Orr (1989), Yumoto (1990), Yumoto et al. (1990), Lin et al. (1991), Yeoman et al. (1991) have all proposed that lowand mid-latitude Pi2 pulsations are driven by compressional cavity resonances. Magnetospheric models predict that cavities should also exist in the outer magnetosphere, although there is little experimental evidence to support this prediction. This may in part be due to greater damping of hydromagnetic waves in the outer magnetosphere compared to the plasmasphere.

Ruohoniemi et al. (1991) and Samson et al. (1992) demonstrated that, on occasions, nightside and earlymorning data from the high-latitude Goose Bay HF radar, Canada, showed structured spectra with distinct 
spectral peaks at a discrete set of frequencies. The frequencies of these spectral peaks were suprisingly stable and decreased with increasing latitude (see Fig. 1).

Samson and co-workers suggested that the spectral peaks were created by field-line resonances driven by magnetospheric waveguide modes. In such a model the frequency of the fundamental resonance and successive harmonics is independent of latitude. Field-line resonances occur when harmonics of the eigenfrequency of the magnetospheric cavity or waveguide match the fundamental eigenfrequency, or successive harmonics, of magnetospheric field lines, resulting in the generation of standing Alfvén waves on the field line at the field-line resonant frequency. Although the explanation offered by Samson et al. (1992) was successful in explaining many of the observed characteristics of these resonances, the mechanism for the stability of the resonant frequencies remains unknown, as magnetospheric cavity and waveguide theory would clearly suggest that the resonant frequencies of the cavity will depend on the dimensions of the magnetospheric cavity, assuming the magnetopause to be the boundary. Such considerations, along with the frequency ratios of the harmonic series, have led to suggestions that the bow shock might be a more appropriate boundary (Allan and McDiarmid, 1993).

Up to the present time such structured spectra have only been detected by the Goose Bay radar. Thus the significance of these observations has been open to debate. Ziesolleck and McDiarmid (1994) employed ground-magnetometer data in search of these monochromatic field-line resonances, but their results proved inconclusive. In view of this a study of the data from the SABRE (Nielsen et al., 1983) radar at Wick has been undertaken to establish whether observations taken from a lower latitude, at a different longitude and with a different radar system would exhibit the same structured spectra.

\section{Observations}

Figure 1 displays power spectra calculated for both the Wick and Goose Bay radars. The Goose Bay spectra were originally calculated by Samson et al. (1992) using the Goose Bay HF radar on 11 January 1989, between 0400 and 0600 LT. The location of the spectral peaks in latitude and frequency have been reproduced here from the published results. No spectral power information is available for these spectra. The Wick spectra have been calculated from 16 days of backscattered power data recorded by the radar between 1981 and 1989, the days being selected because a visual inspection of the data revealed that considerable ULF wave activity was present.

The Wick data employed in Fig. 1 were recorded over a 1-h interval between 0145 and 0245 UT in the latitude range $61{ }^{\circ} \mathrm{N}$ to $67^{\circ} \mathrm{N}$ [altitude-adjusted corrected geomagnetic (AACGM) coordinates, based on PACE geomagnetic coordinates, Baker and Wing, (1989)]. Radar backscattered power data, recorded in $\mathrm{dB}$, were sampled at 20 -s intervals. Thus each interval analysed consisted of 180 data points.

The backscattered power recorded at Wick was filtered at $1000 \mathrm{~s}$ to remove long period variations not associated with ULF wave activity. The data was then reduced to zero mean and any linear trend not associated with ULF wave activity removed, with the time-series then tapered with a $10 \%$ cosine bell. The FFT of the backscattered power was calculated for each of the 16 days. The resulting spectra were averaged in order to highlight any stable spectral features which persisted over the 16 days of the data.

The spectra from the Wick radar have a clear structure. There are three distinct spectral peaks with frequencies of $1.8 \mathrm{mHz}$, a broad region of enhanced spectral power at $2.4-2.8 \mathrm{mHz}$ and a peak at $3.2 \mathrm{mHz}$.

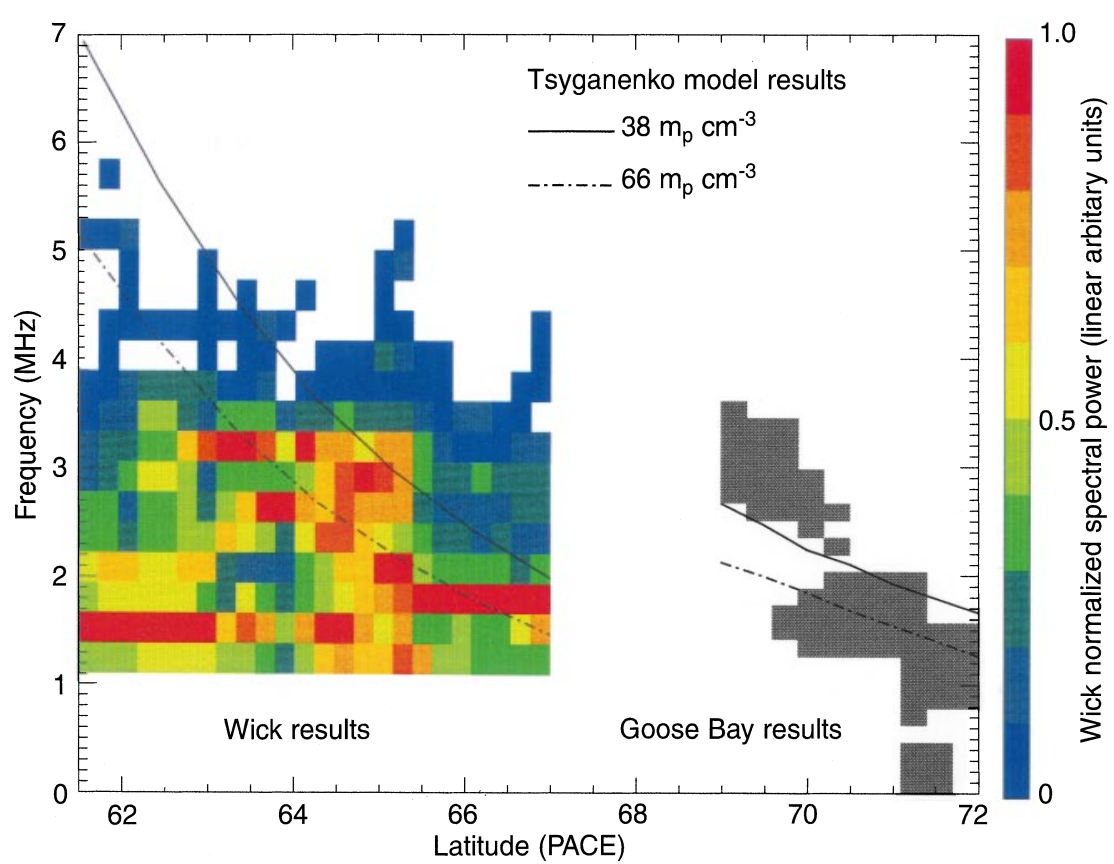

Fig. 1. The latitude dependence of frequency spectra derived from time-series of backscattered power from the Wick VHF coherent radar averaged over 16 days, between 0145 and 0245 UT. The spectral power is normalised to 1 for each latitude bin and is displayed as a linear colour scale with red representing the greatest spectral power. Also displayed are previous results from the Goose Bay radar (Samson et al., 1992, grey shading), and field-line resonance periods for each latitude, estimated from the T89 model for two plasma-density regimes (see text for details). Note the similarity of the frequencies of the spectral peaks for the two instruments 
Although the exact location in frequency of these peaks will be determined by the length and sampling interval of the time-series, as is inevitable in an FFT analysis, the important feature in Fig. 1 is the existence of distinct "hot-spots" in frequency-latitude space, where enhanced ULF wave activity is concentrated. The frequencies of the spectral peaks decrease with increasing latitude, behaviour similar to that observed at Goose Bay. However, the peaks do not appear to belong to a simple harmonic series and there is an additional low-frequency peak of $1.6 \mathrm{mHz}$ at a latitude of $61^{\circ} \mathrm{N}$ which does not fit in with the above quasi-harmonic series.

A comparison of the spectra observed at the Wick and Goose Bay radars reveal that the spectral peaks have a very similar structure. The frequencies of the sequences of field-line resonances at the two radars are almost identical, except for the low-latitude, lowfrequency spectral peak, which is observed only at the Wick radar.

Plotted over the power spectra on Fig. 1 is an estimate of the first harmonic eigenfrequencies of magnetic flux tubes in the magnetosphere. A Tsyganenko magnetic-field model has been employed, with appropriate conditions chosen for the time of the Goose Bay data and appropriate average conditions for the Wick data intervals (see Tsyganenko, 1990). The plasma density was modelled as varying by a factor of $L^{-4}$ in the equatorial plane and by a factor of $R^{-6}$ along the field lines. Here $L$ is the equatorial geocentric distance of the field line measured in Earth radii and $R$ is the radial distance from the centre of the Earth to the field-line element.

Modelling the magnetosphere with an equatorial plasma density equivalent to 66 protons $\mathrm{cm}^{-3}$ at $L=6$ produced a good correlation between the modelled and the observed eigenfrequencies at the Wick radar. A similar plasma density has been inferred before (e.g. Singer et al., 1979; Kivelson et al., 1984) and is likely to represent a particle density of $\sim 10 \mathrm{~cm}^{-3}$ with an effective ion mass of $m i \sim 7 \mathrm{mp}$ (proton masses), caused by the the presence of $\mathrm{O}^{+}$and $\mathrm{He}^{+}$in the plasmatrough. In order to produce a reasonable correlation with the Goose Bay data, an equatorial plasma density equivalent to 38 protons $\mathrm{cm}^{-3}$ at $L=6$ was required.

\section{Discussion}

Observations from the Goose Bay radar have provided evidence of the existence of global magnetospheric cavity/waveguide modes (Samson et al., 1992). Here similar observations from the Wick radar provide independent evidence. The frequency of the fundamental cavity/waveguide resonance and successive harmonics is independent of latitude. Field-line resonances occur when harmonics of the eigenfrequency of the magnetospheric cavity or waveguide match the fundamental eigenfrequency or successive harmonics of resonating geomagnetic field lines, producing an harmonic series of field-line resonances, the frequency of which decreases with increasing latitude. Suprisingly, the eigenfrequency of the cavity/waveguide seems to remain constant and discrete for a number of days.

Average nightside and early-morning data from the SABRE Wick radar show discrete spectra with distinct spectral peaks at 1.8, 2.4-2.8 and $3.2 \mathrm{mHz}$. The results confirm observations of a harmonic series with highly stable frequencies previously made at the Goose Bay HF radar, Canada. At Goose Bay the frequencies of the spectral peaks were $1.3,1.9,2.6-2.7$ and $3.2-3.4 \mathrm{mHz}$. The Wick radar does not detect pulsations with frequencies as low as $1.3 \mathrm{mHz}$. This is due to the radar being at a lower latitude than Goose Bay. The geomagnetic field lines which map into the ionosphere near Wick are too short to support waves of this low frequency. The observations from Wick, derived from a larger database and from a different longitude and latitude sector than those from Goose Bay, and with different instrumentation, have confirmed the Goose Bay observations, and put such radar observations on a more statistical footing.

Although the datasets from the Goose Bay and Wick radars, taken independently, are very similar to magnetospheric cavity resonance/waveguide models, combining the results from the two radar systems has demonstrated the occurrence of two near identical harmonic series at two separate latitudes, in contrast to the expectations from simple cavity-resonance-driven models, which predict that as the latitude decreases the frequency of the spectral peaks increases to match successively higher harmonics of the cavity resonance frequency. The Wick spectra suggest that the frequency of the field-line resonances has a more complex dependence on latitude, and that the first three harmonics of the resonance frequency can be observed in two distinct, latitudinally separated regions.

It is possible that the observed spectra can be explained by only a slight modification of the cavitydriven field-line resonance model, involving a more careful treatment of the effect of the variation of plasma density with $L$-shell on the observed spectra.

If, for example, the field-line resonances observed on the Goose Bay radar are the result of resonances on field lines in the plasmatrough, while the frequencies observed at the lower-latitude Wick radar are created by waves resonating on field lines in the plasmasphere, the increase in ion density in the plasmasphere compared to the plasmatrough would reduce the frequency of Alfvén waves within the plasmasphere. However, an examination of the geomagnetic activity prevailing during the 16 days of Wick data suggests that it is unlikely that the whole of the Wick field of view was in the plasmasphere for a significant number of days.

The plasma density along geomagnetic field lines will vary not only with $L$-shell, but also as a function of time of day and prevailing geomagnetic conditions. As the magnetospheric plasma density varies, the eigenfrequency of a field-line resonance at a given location will fluctuate, or equivalently, the location of a given fieldline resonance frequency will vary. Thus given a cavity resonance frequency which is constant, the latitudinal position of the field-line resonances driven by such a 
cavity resonance will vary, but not the frequency. Such a plasma-density variation could lead to the observation of the identical harmonic series of field-line resonances occurring in distinct latitudinal regions, although a large variation in plasma density is required. An alternative explanation could be that the higher-latitude harmonic series is a consequence of coupling of the same harmonic series of cavity resonances to higher harmonics of the resonant field lines. However, this explanation requires a field-line resonant period of $\sim 1000 \mathrm{~s}$ for the Goose Bay observation at $70^{\circ}-71^{\circ}$ AACGM, which would require an unrealistically high plasma-density distribution, or a very highly distorted geomagnetic field.

Another puzzling feature of the Wick data is the lowlatitude, low-frequency spectral peaks at $1.6 \mathrm{mHz}$. This peak does not fit in with the quasi-harmonic series in the Wick spectra. The modelling work of Allan et al. (1986) and Zhu and Kivelson (1989) suggests that lowfrequency spectral peaks can occur at low latitude if a realistic plasmapause structure is included when modelling the magnetosphere. If the fundamental eigenfrequency of the magnetospheric cavity/waveguide is $\sim 1.3 \mathrm{mHz}$, as suggested by Samson et al. (1992), then $1.6 \mathrm{mHz}$ is a plausible frequency for such a mode (Allan et al., 1986; Zhu and Kivelson, 1989). The low-latitude peak could thus be evidence of such a compressional mode, or could be a field-line resonance driven by the cavity mode within the plasmasphere at the lower latitude of the Wick radar field of view. The frequency of $1.6 \mathrm{mHz}$ is very low for a plasmaspheric field-line resonance, however.

\section{Concluding remarks}

A comparison of the field-line resonances observed at the Goose Bay and Wick radars has been made. Although the two radars cover different latitudinal ranges, the frequencies of the observed field-line resonances are almost identical. In both cases a stable sequence of harmonic peaks was observed. The observed spectra cannot be explained by a straightforward application of the cavity/waveguide resonance theory. Although we believe that the Wick and Goose Bay results point towards the existence of the global magnetospheric cavity modes, much research remains to be done before the detailed structure and source of the modes is understood. A particularly interesting feature is the seeming stability of the cavity/waveguide eigenfrequency in a dynamic magnetosphere.

Further work will include locating the plasmapause for the days of Wick data that have been analysed and a more detailed study of the harmonic structure of the field-line resonances will also be carried out for a substantial number of days. With the CUTLASS radar now working (see Greenwald et al., 1995), it will be possible to bridge the gap between the Goose Bay and Wick radars' fields of view. It appears that a study of the behaviour of the field-line resonances over a wide latitudinal range, as well as consideration of the magnetospheric conditions and structure related to the creation of field-line resonances, is needed before the wave signatures can be fully understood.

Acknowledgments. We would like to thank Dr. John Samson for valuable discussion concerning the Goose Bay field-line-resonance results.

Topical Editor K.-H. Glaßmeier thanks M. Stellmacher and $\mathrm{K}$. Yumoto for their help in evaluating this paper.

\section{References}

Allan, W., and D. R. McDiarmid, Frequency ratios and resonance positions for magnetospheric cavity/waveguide modes, Ann. Geophysical, 11, 916-924, 1993.

Allan, W., E. M. Poulter, and S. P. White, Hydromagnetic wave coupling in magnetosphere-plasmapause effects on impulseexcited resonances, Planet. Space Sci., 34, 1189, 1986.

Baker, K. B. and S. Wing, A new magnetic coordinate system for conjugate studies at high latitude, J. Geophys. Res., 94, 91399144, 1989.

Chen, L., and A. Hasegawa, A theory of long period magnetic pulsation 1. steady state excitation of a field line resonance, J. Geophys. Res., 79, 1024-1032, 1974.

Dungey, J. W., Electrodynamics of the outer atmosphere, Penn. State Univ. Ionos. Res. Lab. Sci. Rep., 69, 1954.

Greenwald, R. A., K. B. Baker, J. R. Dudeney, M. Pinnock, T. B. Jones, E. C. Thomas, J.-P. Villain, J.-C. Cerisier, C. Senior, C. Hanuise, R. D. Hunsucker, G. Sofko, J. Koehler, E. Nielsen, R. Pellinen, A. D. M. Walker, N. Sato and H. Yamagishi, Darn/ Superdarn: A global view of the dynamic of high-latitude convection, Space Sci. Rev., 71, 761-796, 1995.

Hughes, W. J., Hydromagnetic waves in the magnetosphere, in Solar-Terrestrial Physics, Eds. R. L. Carovillano and J. M. Forbes, D. Reidel, Dortrecht, 1983.

Kivelson, M. G., and D. J. Southwood, Resonant ULF waves: A new interpretation, Geophys. Res. Lett., 12, 49-52, 1985.

Kivelson, M. G., J. Etcheto, and J. G. Trotignon, Global compressional oscillations of the terrestrial magnetosphere: the evidence and a model, J. Geophys. Res., 89, 9851-9856, 1984.

Lin, C. A., L. C. Lee and Y. J. Sun, Observation of Pi2 pulsations at very low latitude $(\mathrm{L}=1.06)$ station and magnetospheric cavity resonances, J. Geophys. Res., 96, 21105-21113, 1991.

Nielsen, E., W. Guttler, E. C. Thomas, C. P. Stewart, T. B. Jones, and A. Hedburg, SABRE - A new radar auroral backscatter experiment, Nature, 304, 712-714, 1983.

Ruohoniemi, J. M., R. A. Greenwald, K. B. Baker, and J. C. Samson, HF radar observations of Pc5 field line resonances in the midnight/early morning MLT sector, J. Geophys. Res., 96, 15697-15710, 1991.

Samson, J. C., B. G. Harrold, J. M. Ruohoniemi, R. A. Greenwald and A. D. M. Walker, Field line resonances associated with MHD waveguides in the magnetosphere, Geophys. Res. Lett., 19, 441-444, 1992.

Singer, H. J., C. T. Russell, M. G. Kivelson, T. A. Fritz, and W. Lennartson, Satellite observations of the spatial extent and structure of $\mathrm{Pc} 3,4,5$ pulsations near the magnetospheric equator, Geophys. Res. Lett., 6, 889-892, 1979.

Southwood, D. J., Some features of field line resonances in the magnetosphere, Planet. Space Sci., 22, 483-491, 1974

Sutcliffe, P. R., and K. Yumoto, Dayside Pi2 pulsations at low latitude, Geophys. Res. Lett., 16, 887-890, 1989.

Sutcliffe, P. R. and K. Yumoto, On the cavity mode nature of low-latitude Pi2 pulsations, J. Geophys. Res., 96, 1543-1551, 1991.

Tamao, T., Transmission and coupling resonance of hydromagnetic disturbances in the non-uniform Earth's magnetosphere, Sci. Rep. Tohoku Univ. series 5, Geophys., 17, 43-72, 1965. 
Tsyganenko, N. A., Quantitative models of the magnetospheric magnetic field: methods and results, Space Sci. Rev., 54, 75-186, 1990.

Yeoman, T. K. and D. Orr, Phase and spectral power of midlatitude Pi2 pulsations: evidence for a plasmaspheric cavity resonance, Planet. Space Sci., 37, 1367-1383, 1989.

Yeoman, T. K., M. Lester, D. K. Milling, and D. Orr, Polarization, propagation and MHD wave modes of $\mathrm{Pi} 2$ pulsations: SABRE/ SAMNET results, Planet. Space Sci., 39, 983-998, 1991.

Yumoto, K., Evidence of magnetospheric cavity Pi2 waves, J. Geomagn. Geoelectr., 42, 1281-1290, 1990.
Yumoto, K., K. Takahashni, T. Sakurai, P. R. Sutcliffe, S. Kokubun, H. Lühr, T. Saito, M. Kuwashima and N. Sato, Multiple groundbased and satellite observations of global Pi2 magnetic pulsations,J. Geophys. Res., 95, 15175-15184, 1990.

Zhu, X., and M. G. Kivelson, Global mode ULF pulsations in a magnetosphere with a non-monotonic Alfvén velocity profile, J. Geophys. Res., 94, 1479-1485, 1989.

Ziesolleck, C. W.S, and D. R. McDiarmid, Auroral latitude Pc5 field line resonances: quantized frequencies, spatial characteristics and diurnal variation, J. Geophys. Res., 99, 5817-5830, 1994. 